\title{
ON GENERALIZATIONS OF HOPF'S CLASSIFICATION THEOREMS
}

\author{
HiRoshi MiYaZAKAI
}

(Received December 24, 1952)

Hopf's brilliant results have been generalized to various general cases by H. Whitney [13] ${ }^{1)}$, P.Alexandroff [1], S. Eilenberg [3]. C. H. Dowker [2] and S. T. Hu [4].

The purpose of this paper is to generalize the Hu's work [4] and to obtain Hopf's classification theorems which contain the results of Dowker [2] and $\mathrm{Hy}$ [4].

1. Fúndamental Bridge Theorems.

In this section we shall provide Hu's fundamental bridge theorems in more general forms which are the main tools of his paper [4]. Therefore the majority of his results in [4] will be generalized.

Let $X$ be a normal space and $X_{0}$ be its closed subset. By a covering [finite covering] $\alpha$ of $X$ we shall always means a locally finite [finite] open covering of $X$. The nerve of $\alpha$ with the weak topology (see [8, Definition $3.1]$ ) is denoted by $A$. The nerve $A_{0}$ of the covering $\alpha \cap X_{0}^{2)}$ may be regarded as a subcomplex of $A$. Let denote a canonical mapping (see [8, Definition 3.2]) of $\left(X, X_{0}\right)$ into $\left(A, A_{0}\right)$. Since $X$ is normal and coverings considered are locally finitet, here always exist canonical mappings [7,, Lemma 2].

Let $f$ be a given mapping of $X_{0}$ into an arbitrary space $Y$ and $\alpha$ a covering of $X$. A mapping $\psi_{\alpha}: A_{0} \rightarrow Y$ is called a bridge mapping for $f$, if the partial mapping $\psi_{a} \phi_{a} \mid X_{0}$ is homotopic to $f$ for each canonical mapping $\phi_{\alpha}:\left(X, X_{0}\right) \rightarrow\left(A, A_{0}\right)$. If such a bridge mapping exists, $\alpha$ is said to be a bridge for the mapping $f$. In particular, if $Y$ is compact and $\alpha$ is a finite covering and $\psi_{\alpha} \phi_{\alpha} \mid X_{0}$ is uniformly homotopic [references $7_{1}, \S 2$, p. 86] to $f$, then $\psi_{\alpha}$ and $\alpha$ are called a finite bridge mapping and a finite bridge for $f$ respectively.

Bridge Refinement Theorem. For a given mapping $f: X_{0} \rightarrow Y$, any locally finite refinement $\beta$ of a bridge $\alpha$ is a bridge.

Proof. Let $\psi_{\alpha}: A_{0} \rightarrow Y$ be a bridge mapping for $f ; \phi_{\alpha}:\left(X, X_{0}\right) \rightarrow\left(A, A_{0}\right)$ be an arbitrary canonical mapping. Since $\beta$ is a refinement of $\alpha$ there exists a simplicial projection $p_{\beta \alpha}:\left(B, B_{0}\right) \rightarrow\left(A, A_{0}\right)[8, \S 9]$. By the first half of Corollary $9.3[8], \phi_{\alpha} \simeq p_{\beta \alpha} \phi_{\beta}$. Hence

$$
f \simeq \psi_{\alpha} \phi_{\alpha}\left|X_{0} \simeq \psi_{\alpha} p_{\beta \alpha} \phi_{\beta}\right| X_{0} .
$$

Thus $\beta$ is a bridge and $\psi_{\alpha} p_{\beta \alpha}: B_{0} \rightarrow Y$ is a brige mapping for $f$.

1) Numbers in square brackets refer to the references cited at the end of this paper.

2) $\alpha \cap X_{0}$ means a covering of $X_{0}$ consisting of all intersections of $X_{0}$ and elements of $\alpha$. 
Finite Bridge Refinement Theorem. Let $Y$ be a compact space. For a given mapping : $X_{0} \rightarrow Y$, any finite refinement $\beta$ of a finite bridge $\alpha$ is a finite bridge.

PRoof. Let $\psi_{\alpha}, \phi_{\alpha}$ and $p_{\beta \alpha}$ be the same as in the preceding theorem. In the present case $A$ and $B$ are both finite complexes. By the second part of Corollary $9.3[8], \phi_{\alpha} \stackrel{\imath}{\simeq} p_{\beta \alpha} \phi_{\beta}$, hence we have

$$
f \stackrel{w}{\simeq} \psi_{\alpha} \phi_{\alpha}\left|X_{0}, \stackrel{u}{\simeq} \psi_{\alpha} p_{\beta \alpha} \phi_{\beta}\right| X_{0} .
$$

Thus $\beta$ is a finite bridge with a bridge mapping $\psi_{\alpha} p_{\beta \alpha}$.

BRIDGe Existence Theorem. If $Y$ is dominated ${ }^{3)}$ by a $C W$-complex ${ }^{4}$, every mapping $f: X_{0} \rightarrow Y$ has a bridge.

Finite BRIDge Existence Theorem. If $Y$ is compact and is dominated by a CW-complex, then every mapping $f: X_{0} \rightarrow Y$ has a finite bridge.

Proof. Any CW-complex is of the same homotopy type as its singular complex [12, Theorem 23] and the singular complex of a space has a simplicial decomposition $[5,(7.1)$, p.172]. Hence $Y$ is dominated by a simplex. Furthermore, if $Y$ is compact then, $Y$ is dominated by a finite simplicial complex [12, Appendix A, p.107].

Let $\lambda: Y \rightarrow P$ and $\mu: P \rightarrow Y$ be mappings such that $\mu \lambda \simeq 1$, where $P$ is a simplicial complex, and in the case where $Y$ is compact, $P$ is finite simplicial complex. Let $\left\{s t p_{j}\right\}$ be the covering consisting of all open stars of vertices $p_{j} \in P$, and set $\alpha_{0}^{\prime}=\left\{(\lambda f)^{-1}\left(s t p_{j}\right)\right\}$. Then $\alpha_{0}^{\prime}$ is a covering of $A_{0}$ and if $Y$ is compact, then $\alpha_{0}^{\prime}$ is a finite covering. Let $\alpha=\left\{a_{0}, a_{j}\right\}$ be a family of open sets in $X$ such that $a_{0}=X-X_{0}, a_{j} \cap X=(\lambda f)^{-1}\left(s t p_{j}\right)$. Let $\phi^{\alpha}:(X$, $\left.\mathrm{X}_{0}\right) \rightarrow\left(A, A_{0}\right)$ be a canonical mapping and $\tau$ the simplicial mapping defined by the vertices correpsondence $a_{j} \rightarrow p_{j}$. Since $\phi_{a} \mid X_{0}$ is a canonical mapping of $X_{0}$ into the nerve $A_{0}$ of the covering $\alpha \cap X_{0}$, by Lemma 3.4 [8], $\lambda f \simeq$ $\tau \phi_{\alpha} \mid X_{0}$ and if $A$ is finite then $\lambda f \stackrel{u}{\simeq} \tau \phi_{a} \mid X_{0}$. Hence $f \simeq \mu \lambda f \simeq \mu \tau \phi_{a} \mid X_{0}$, and if $Y$ is compact and $P$ is finite then $f \stackrel{u}{\simeq} \mu \lambda f \stackrel{u}{\simeq} \mu \tau \phi_{\alpha} \mid X_{0}$ because $\mu \lambda$ is uniformly continuous. Thus $\alpha$ and $\psi_{\alpha}=\mu \tau$ are a bridge and a bridge mapping for $f$ and in the case where $Y$ is compact, these are a finite bridge and a finite bridge mapping for $f$. Thus the above two theorems are established.

BRIDGe Homotopy THEOREM. Let $X$ be a normal space and $X_{0}$ its closed paracompact ${ }^{5}$ space. Let $\alpha$ and $\beta$ be two bridges for a given mapping $f$ of $X_{0}$ into a space $Y$ which is dominated by a $C W$-complex, and let $\psi_{a}: A_{0} \rightarrow Y$, $\psi_{\beta}: B_{0} \rightarrow Y$ be bridge mappings. Then there exists a common refinement

3) See $[11$, p. 214].

4) See $[11, \S 5$, p. 223].

5) A topological space $X_{0}$ is said to be paracompact if any open covering (not necessary locally finite) of $X$ has a locally finite refinement. 
$\gamma$ of $\alpha$ and $\beta$ such that $\psi_{\alpha \alpha} p_{\gamma \alpha} \mid C_{0}$ and $\psi_{\beta} b_{\gamma \beta} \mid C_{0}$ are homotopic, where $p_{\gamma \alpha}$ : $\left(C, C_{0}\right) \rightarrow\left(A, A_{0}\right), p_{\gamma \beta} ;\left(C, C_{0}\right) \rightarrow\left(B, B_{0}\right)$ are arbitrary projections.

Proof. By the hypothesis, there exist a simplicial complex $P$ and mappings $\lambda: Y \rightarrow P, \mu: P \rightarrow Y$ such that $\mu \lambda \simeq 1$. Let us put $g=\lambda f, \psi_{a}^{\prime}=\lambda \psi_{\alpha}$, $\psi_{\beta}^{\prime}=\lambda \psi_{\beta}$. Since $\psi_{\alpha}, \psi_{\beta}$ are bridge mappings for $f$, we have

$$
\psi_{\alpha}^{\prime} \phi_{\alpha} \mid X_{0} \simeq g \simeq \psi_{\beta}^{\prime} \phi_{\beta} X_{0} .
$$

By the proof of the first half of Theorem 9.4 [8], it is easily seen that there exists a common refinement $\gamma$ of $\alpha$ and $\beta$ such that

$$
\psi_{\alpha}^{\prime} p_{\gamma \alpha}\left|C_{0} \simeq \psi_{\beta}^{\prime} p_{\gamma 3}\right| C_{0} .
$$

Hence

$$
\begin{aligned}
& \psi_{\alpha} p_{\gamma \alpha}\left|C_{0} \simeq \mu \lambda \psi_{\alpha} p_{\gamma \alpha}\right| C_{0}=\mu \psi_{\alpha}^{\prime} p_{\gamma \alpha} \mid C_{0} \\
\simeq & \mu \psi_{\beta}^{\prime} p_{\gamma \beta}\left|C_{0}=\mu \lambda \psi_{\beta} p_{\gamma \beta}\right| C_{0} \simeq \psi_{\beta} p_{\gamma \beta} \mid C_{0} .
\end{aligned}
$$

This completes the proof.

Finite Bridge Homotopy Theorem. Let $X$ be a normal space and $\mathrm{X}_{0}$ its closed subset. Let $\alpha$ and $\beta$ be two finite bridges for a given mapping $f$ of $X_{0}$ into a compact normal space $Y$ which is dominated by a CW-complex, and let $\psi_{\alpha}: A_{0} \rightarrow Y, \psi_{\beta}: B_{0} \rightarrow Y$, be finite bridge mappings. Then there exists a common finite refinement $\gamma$ of $\alpha$ and $\beta$ such that $\psi_{\alpha} p_{\gamma \alpha} \mid C_{0}$ and $\psi_{\beta} p_{\gamma \beta} \mid C_{0}$ are uniformly homotopic, where $p_{\gamma \alpha}:\left(C, C_{0}\right) \rightarrow\left(A, A_{0}\right), p_{\gamma \beta}:\left(C, C_{0}\right) \rightarrow\left(B, B_{0}\right)$ are arbitrary projections.

Proof. By the hypothesis, there exist a finite simplicial complex $P$ and mappings $\lambda: Y \rightarrow P, \mu: P \rightarrow Y$ such that $\mu \lambda \simeq 1$. Since $P$ and $Y$ are compact we know that $\mu \lambda \stackrel{u}{\simeq} 1$. Since $\psi_{\alpha}, \psi_{\beta}$ are finite bridge mappings for $f$, and $\lambda$ is uniformly continuous, we have

$$
\psi_{\alpha}^{\prime} \phi_{\alpha}\left|X_{0} \stackrel{u}{\simeq} g \simeq \psi_{\beta}^{\prime} \phi_{\beta}\right| X_{0} .
$$

where $g=\lambda f, \quad \psi_{\alpha}^{\prime}=\lambda \psi_{\alpha}, \quad \psi_{\beta}^{\prime}=\lambda \psi_{\beta}$.

By the proof of the second half of Theorem $9.4[8]$, there exists a common finite refinement $\gamma$ of $\alpha$ and $\beta$ such that

Hence we have

$$
\psi_{\alpha}^{\prime} p_{\alpha}^{\gamma}\left|C_{0} \stackrel{u}{\simeq} \psi_{\beta}^{\prime} p_{\gamma \beta}\right| C_{0} .
$$

This completes the proof.

$$
\psi_{\alpha} p_{\gamma \alpha}\left|C_{0} \stackrel{n}{\simeq} \psi_{\beta} p_{\gamma \beta}\right| C_{0}
$$

\section{Hopf's Classification Theorems.}

In this section we shall assume that $Y$ is a connected space dominated by a CW-complex and satisfying $\pi_{r}(Y)=0$ for each $1 \leqq r \leqq n$, where $\pi_{r}(Y)$ denotes $r^{\text {th }}$ homotpoy group of $Y$. If $n>1$, the latter condition implies $\pi_{1}(Y)=0$ and hence the $i$-simplicity of $Y$ for all $i$. If $n=1$ we assume the $i$-simplicity of $Y$ for each $i \leqq m$, where $m$ is an integer to be specified in the sequel. 
Let $\alpha$ be an arbitrary bridge for $f: X \rightarrow Y$ with $\psi_{\alpha}: A \rightarrow Y$ as a bridge mapping. Since $\pi_{r}(Y)=0$ for each $r<n$, we may assume that $\psi_{\alpha}\left(A^{n-1}\right)=$ $y_{0}$, where $A^{q}$ denotes the $q$-skeleton of $A$ and $y_{0}$ is a fixed point in $Y$. For each oriented $n$-simplex $\sigma_{i}^{n} \in A$, the partial mapping $\psi_{a} \mid \sigma_{i}^{n}$ determines an element $\left(\psi_{a}, \sigma_{i}^{n}\right)$ of homotopy group $\pi_{n a}(Y)$. Since $\psi_{a}$ is defined throughout $A$, the $n$-cochain

is clearly a cocyle of $A$.

$$
k^{n}\left(\psi_{\alpha}\right)=\Sigma\left(\psi_{a \alpha}, \sigma_{i}^{n}\right) \sigma_{i}^{n}
$$

By the same way as in the proof of (9.1) [4, p.353], we know that all the possible cocycles $k^{n}\left(\psi_{\alpha}\right)$ represent a unique element $\kappa^{n}(f)$ of $H^{n}\left(X, \pi_{n}(Y)\right)^{6}$, where we assumed that $X$ is paracompact.

If $Y$ is a compact normal space we restrict ourself to all finite bridges $\alpha$ and finite bridge mappings $\psi_{\alpha}$, then it is also seen that all the possible cocycles $k^{n}\left(\psi^{\alpha}\right)$ represent a unique element $\kappa_{F}^{n}(f)$ of $\left.H_{F}^{n}\left(X, \pi_{n}(Y)\right)^{7}\right)$. In this case we do not assume the paracompactness of $X$. The elements $\kappa^{n}(f)$ and $\kappa_{F}^{n}(f)$ are both called the characteristic elements of $f$.

Now we can state the following generalized Hopf's classification theorems.

THEOREM I. If $X$ is a paracompact normal space with dim $X \leqq m^{\S)}$ and $H^{r}\left(X, \pi_{r}(Y)\right)=0=H^{r+1}\left(X, \pi_{r}(Y)\right)$ for each $n<r \leqq m$, then the elements of $H^{n}\left(X, \pi_{n}(Y)\right)$ are in $a(1-1)$-correspondence with the homotopy classes of the mappings $f: X \rightarrow Y$. The correspondence is determined by the operation $\kappa^{n}(f)$.

THEOREM II. If $X$ is a normal space with $\operatorname{dim} X \leqq n$, and $H^{r}(X, \pi(Y))=$ $0=H^{r+1}\left(X, \pi_{r}(Y)\right)$ for each $n<r \leqq m$, then the elements of $H^{\prime \prime}\left(X, \pi_{n}(Y)\right)$ are in a $(1-1)$-correspondence with the uniform homotopy classes of the mapping $f: X \rightarrow Y$. The correspondence is deiermined by the operation $\kappa_{F}^{n}(f)$, where $Y$ is compact.

If $Y$ is paracompact and normal, then the characteristic element $\kappa^{i n}(\tau) \in$ $H^{\prime \prime}\left(Y, \pi_{n}(Y)\right)$ of the identity mapping $\tau: Y \rightarrow Y$ can be considered. And if $Y$ is compact, $\left.\kappa_{F(}^{n} \tau\right)$ may be regarded as an element of $H_{F}^{n}\left(Y, \pi_{n}(Y)\right)$ $\left(\approx H^{n}\left(Y, \pi_{n}(Y)\right)\right)$.

Theorem. I'. If $X$ is a paracompact normal space with dim $X \leqq n$ and $\pi(Y)=0$ except $n$, then the homotopy classes of mappings $f: X \rightarrow Y$ are in a (1-1)-correspondence with the group $H^{n}\left(X, \pi_{n}(Y)\right)$. The correspondence is determined by the operation $f \rightarrow f^{*}\left(\kappa^{n}(\tau)\right)$, where $f^{*}: H^{n}\left(Y, \pi_{n}(Y)\right)$ is the homomorphism induced by the mapping $f$.

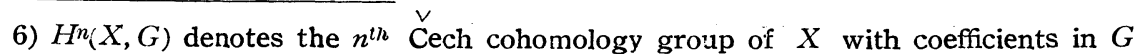
based on all open cpverings (not necessary locally finite) of $X$. See $[2, \S 4$, p. 213].

7) $H_{H^{\prime}}^{n}(X, G)$ denotes the $n$th $\stackrel{\vee}{C}$ ch cohomology group of $X$ with coefficients in $G$ based on all finite open coverings of $X$.

8) For the definition on $\operatorname{dim} X$ see $[2, \S 3$, p. 206]. 
THEOREM II'. If $X$ is a normal space with $\operatorname{dim} X \leqq n, Y$ is a compact normal space and $\pi_{r}(Y)=0$ except $n$, then the uniform homootpy c'asses of mappings $f: X \rightarrow Y$ are in $a(1-1)$-correspondence with the group $H^{n}\left(X, \pi_{n}(Y)\right)$. The correspondence is determined by the operation $f \rightarrow f^{* F}\left(\kappa^{n}(\tau)\right)$, where $f^{* F}$ : $H^{n}\left(Y, \pi_{n}(Y)\right) \rightarrow H^{n}\left(X, \pi_{n}(Y)\right)$ is the homomorphism induced by the mapping $f$.

These Theorems I, II, I' and II' $^{\prime}$ can be easily obtained by the same arguments as in [4] if we use our fundamental bridge and finite brige theorems instead of Hu's fundamental bridge theorems and using of Theorem 3.5 [2]. And so we shall omit the complete proof.

REMARK. Theorems $\mathrm{I}^{\prime}$ and $\mathrm{II}^{\prime}$ contain Theorem 7.5 and 9.3 [2] as special cases. It is immediately follows by a theorem proved in Appendix that our theorem $\mathrm{I}^{\prime}$, II, $\mathrm{I}^{\prime}$ and $\mathrm{II}^{\prime}$ are generalizations of the Hu's results $[4, \mathrm{p}$. 356]. We shall also notice that the majority of Hu's work [4] can be generalized in our general cases.

\section{APPENDix}

A metric [separable metric] space $Y$ is said to be a ANR [separable ANR] if for any metric [separable metric] space $Z$ which contains $Y$ as its closed subset, $Y$ is a neighborhood retract ${ }^{9}$ ) of $Z$. We shall prove the following theorem ${ }^{10)}$

Theorem. If $Y$ is a ANR or separable ANR then $Y$ is dominated by a simplicial complex with the weak topology.

Proof. According to a theorem due to Wojdyslawski $[14$, p. 186], $Y$ can be imbedded as a closed set of a convex subset $Z$ of a Banach space $W$, and $W$ is separable when $Y$ is separable. Since $Y$ is ANR [or separable ANR], there exist an open set $V$ of $Z$ containing $Y$ and a retraction $\theta: V \rightarrow Y$. For each point $y \in Y$, let $S(y)$ denote an open spherical neighborhood of $y$ in $Z$ such that $S(y) \subset V$. Since $Z$ is convex, $S(y)$ is also convex. $Y$ is metric and hence paracompact ${ }^{11}$. Therefore there exists a locally finite open covering $\left\{U_{\alpha}\right\}$ of $Y$ which is a refinement of $\{S(y) \cap Y\}$. Let $K$ be the nerve of the covering $\left\{U_{a}\right\}$ and let $u$ denote the vertex corresponding to a element $U_{a}$. For a finite points $y_{0}, \ldots, y_{n} \in Y$ let $\left[y_{0}, \ldots, y_{n}\right]$ denote the minimal convex set containing $y_{0}, \ldots, y_{n}$ in $W$. Hence $\left[y_{0}, \ldots, y_{n}\right]$ consists of points $a_{0} y_{0}+\ldots+a_{n} y_{n}$, where $a_{0}, \ldots, a_{n}$ are non-negative real numbers such that $a_{0}+\ldots+a_{n}$ $=1$.

For each vertex $u_{\alpha} \in K$, let us choose a point $y_{\alpha} \in Y$ such that $U_{\alpha} \subset S\left(y_{\alpha}\right)$.

9) See $[6, \S 5$, p. 58].

10) This theorem for compact separable ANR is well-known. See [6, Theorems 12.2, 16. 2 , pp. 93, 99].

11) See $[9$, Theorem 1] and [10, Theorem 8,14, p. 53]. 
For each $n$-simplex $\sigma=\left(u_{\alpha_{0}}, \ldots, u_{\alpha_{n}}\right) \subset K$ we define a mapping $\nu_{\sigma}: \bar{\sigma} \rightarrow$ $\left[y_{\alpha_{0}}, \ldots, y_{\alpha_{n}}\right]$ by taking $\nu_{\sigma}(x) a_{0} y_{\alpha_{0}}+\ldots+a_{n} y_{\alpha_{n}}(x \in \bar{\sigma})$, where $a_{0}, \ldots a_{n}$ are barycentric coordinates of $x$ with respect to the vertices $u_{\alpha_{0}}, \ldots, u_{a_{n}}$. Then $\nu_{\sigma}$ define a continuous mapping

$$
\nu: K \rightarrow W .
$$

If $u_{\alpha_{0}}, \ldots, u_{\alpha_{n}}$ are vertices of a simplex in $K$, then $U_{\alpha_{0}} \cap \ldots \cap U_{\alpha_{n}} \neq 0$, hence $S\left(y_{\alpha_{0}}\right) \cap \ldots \cap S\left(y_{\alpha_{n}}\right) \neq 0$. Hence $S\left(y_{\alpha_{\alpha}}\right) \cup \ldots \cup S\left(y_{\alpha_{n}}\right) \supset\left[y_{\alpha_{0}}, \ldots, y_{\alpha_{n}}\right]$. Therefore $\nu(K) \subset V$.

We put $\mu=\theta \nu$. Let $\lambda: Y \rightarrow K$ be a canonical mapping. It is remains to show that $\mu \lambda \simeq 1$.

For each point $y \in Y$ let $U_{\alpha_{0}}, \ldots, U_{\alpha_{n}}$ be all elements of $\left\{U_{\alpha}\right\}$ which

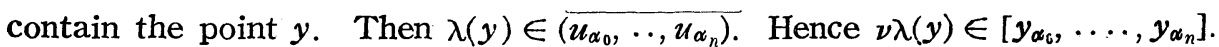
On the other hand $y \in U_{\alpha_{0}} \cap \ldots \cap U_{a_{n}} \subset S\left(y_{\alpha_{0}}\right) \cap \ldots \cap S\left(y_{\alpha_{n}}\right)$. Hence a point $\rho_{t}(y)$ which divides the segment joining $\nu \lambda(y)$ and $y$ into a ratio $1-t: t(0 \leqq t$ $\leqq 1$ ) always belongs to som $S\left(y_{\alpha_{j}}\right)$ and hence belongs to $V$. Hence

$$
\xi_{t}=\theta \rho_{i}: Y \rightarrow Y
$$

is a well-defined homotcpy between $\xi_{\iota}(y)=\epsilon \rho_{\iota}(y)=\epsilon \nu \lambda(y)$ and $\xi_{0}(y)=\theta \rho_{0}(y$ $=\theta(y)=y$. Hence $\mu \lambda \simeq 1$. This completes the proof.

\section{REFERENCES}

1 P. Alexandroff, On the dimension of normal spaces, Proc. Royal Soc. Ser. A. vol. 189(1947), 11-39.

2 C. H. DOWKER, Mapping theorems for non-compact spaces, Amer. Journ. of Math., vol. 69(1947), 200-242.

3 S. EIlenberG, Cohomology and continuous mappings, Ann. of Math. vol. 41(1940), 231-252

4 S. T. HU, iMappings of a normal space into an absolute neighborhood retract, Trans. Amer. Math. Soc., vol. 64(1948), 336-358.

5 S. T.HU, Extensions and classifications of mappings, Osaka Math. Jour. vol. 2(1950), 165-209.

6 S. LeFsCHETZ, Topics in topology, Princeton, 1942.

7 H. MIYAZAKI, On the covering homotopy theorems, Tôhoku Math. Jour. Vol. 4(1952). 80-87.

72 H. MIYAZAK!, A note on paracompact spaces Tohoku Math. Jour. vol. 4(1952), 88-92.

8 H. MIYAZAII, The cohomotopy and uniform cohomotopy groups, Tohoku Math. Jour. Vol 5.

9 A. H. STONE, Paracompactness and pioduct spaces, Bull. Amer. Math. Soc., Vol. 54 (1948), 977-982.

10 J. W. TUKEY, Convergence and uniformity in topology, Princeton, (1940).

11 J. H. C. WHITEHEAD, Combinatorial homotopy I, Bull. Amer. Math. Soc. Vol. 55(1949), 213-245.

12 J. H. C. WHITEHEAD, A certain exact sequence, Ann. of Math. Vol. 52(1950), 51-108.

13 H. WhITNEY, The maps of an $n$-complex into an $n$-sphere, Duke Math, Journ. Vol. 3(1937), 51-55.

14 M. WOJODYSLAWSKI, Retractes absolus et hyperespaces des continus, Fund. Math. Vol. 32(1939), 184-192.

MATHEMATICAL INSTITUTE, TÔHOKU UNIVERSITY. 